\title{
Delays in the referral and primary management of cutaneous malignant melanoma at Tygerberg Hospital
}

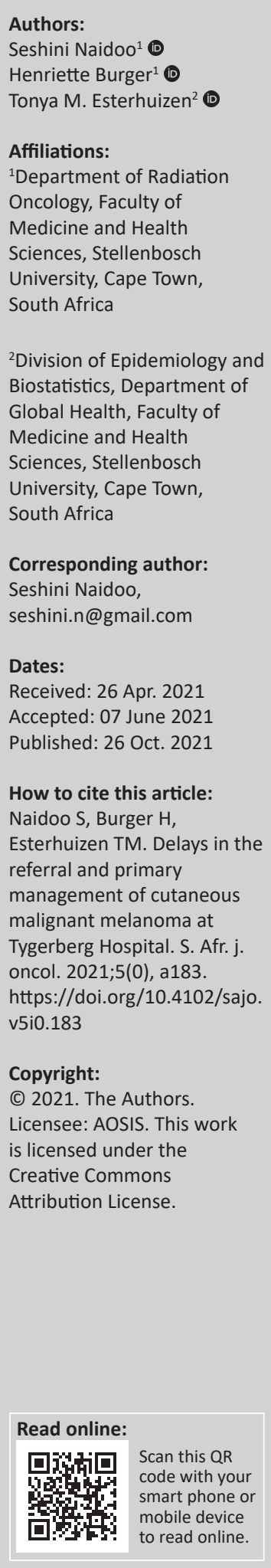

Background: Cutaneous malignant melanoma (CMM) is a significant cause of skin cancerrelated mortality. The time between the diagnostic biopsy and primary surgical excision, the surgical interval (SI), is a modifiable factor that may impact melanoma outcomes. Delays in the SI are attributable to many factors.

Aim: To determine the SI in patients with resectable CMM treated at Tygerberg Academic Hospital (TAH).

Methods: A retrospective review of patients referred to the TAH multidisciplinary melanoma clinic with histologically confirmed CMM between January 2015 and December 2017 was done. Patients $>18$ years with resectable melanoma (T1b-T4b N0-3 M0-1a) who received definitive surgery were included.

Results: The cohort $(n=40)$ comprised mostly Caucasians referred from the Cape metropolitan (metro) area, with a median age at diagnosis of 59 years. Thirty-one (77.5\%) patients had T3 or T4 lesions on diagnostic biopsy. Twenty patients (50\%) underwent a sentinel lymph node biopsy (SLNB) which led to an upstaging in $20 \%$ of cases. The median length of the SI was 13.5 weeks. Eighteen patients (45\%) underwent primary excision within the recommended 12 weeks from diagnostic biopsy. There was a significant association between the SI and patients living in the Cape metro $(p=0.04)$ as well as the SI and p Stage $(p=0.01)$.

Conclusion: Surgical interval guidelines for cutaneous melanoma are poorly defined. We used 12 weeks as an extrapolation of international guidelines. Even though this target was not met, the study is proposed to be of value in guiding future protocols and ultimately allowing for improved, timely service to patients.

Keywords: surgical interval; melanoma; biopsy; delay; skin cancer.

\section{Introduction}

Cutaneous malignant melanoma (CMM) represents 3\% of all skin cancer diagnoses; however, it is responsible for $65 \%$ of skin cancer-related deaths worldwide. ${ }^{1}$ According to the National Cancer Institute and Surveillance, Epidemiology, and End Results Program (SEER) data, it is the second most common cancer amongst adolescents and adults. ${ }^{2}$ The incidence continues to increase dramatically at an overall rate of 33\% for men and 23\% for women from 2002 to 2006 in the United States (US). ${ }^{3}$

The South African Caucasian population has one of the highest incidences of CMM in the world. The exposure to year-round high ambient solar ultraviolet radiation and latitude $\left(22^{\circ} \mathrm{S}-34^{\circ} \mathrm{S}\right)$ makes these South Africans, especially susceptible to skin cancer. The estimated yearly incidence of CMM in South Africa is 4.76 per 100000 persons overall and 19.2 per 100000 in Caucasians. In 2009, the Western Cape Province of South Africa's melanoma incidence for Caucasians was unofficially reported as high as 69 per 100000 people. $^{4}$

Cutaneous malignant melanoma diagnosed early as a localised disease with a Breslow tumour thickness of $\leq 1 \mathrm{~mm}$ has an excellent prognosis with a 5 year survival rate of $>90 \%$. In patients with localised melanomas $>1.0 \mathrm{~mm}$ in thickness, 5 year survival rates range from $50 \%$ to $90 \%$, depending on tumour thickness, ulceration and mitotic rate. ${ }^{3}$ Cutaneous malignant melanoma has the highest rate of regional and distant metastases amongst skin cancers. ${ }^{5}$ Distant metastatic disease is associated with an inferior prognosis ${ }^{2,3}$; thus early diagnosis and treatment of CMM are considered paramount, and delays in treatment are hypothesised to affect survival. ${ }^{6}$ 
Access to newer treatments that offer a survival advantage in advanced and metastatic melanoma is restricted in resourcelimited settings; therefore, early diagnosis and appropriate, timely surgical treatment are the primary recourse in the South African public healthcare system.

Surgical excision remains the primary treatment for CMM. The standard process for diagnosing and treating malignant melanoma involves a two-step procedure with an initial diagnostic biopsy followed by a more definitive excision with wide surgical margins. ${ }^{6}$

The time between the diagnostic biopsy and definitive surgery is the surgical interval (SI). Delays in the SI are attributable to many factors related to the patient, the disease and the healthcare system. Such delays may evoke psychological stress and anxiety in patients, even though the impact on morbidity and mortality remains unclear. Existing literature is yet to provide a consensus on the optimal SI.7,8

This study's objectives were to determine the magnitude of the SI in patients with localised CMM treated at TAH, describe factors associated with delays, identify associations between descriptive variables and the SI and estimate the cohort's overall survival (OS).

The findings motivate for public awareness, education and improved screening programmes in melanoma. The factors associated with treatment and referral delays can be critically reviewed to improve and streamline service provision.

\section{Methodology}

A retrospective chart review of histologically confirmed localised CMM was conducted at the Division of Clinical and Radiation Oncology, TAH.

Tygerberg Academic Hospital, a tertiary referral hospital in the Western Cape, runs a weekly melanoma multidisciplinary team meeting (MDT), where management decisions are made for all newly diagnosed $\mathrm{T} 1 \mathrm{~b}$ and above melanomas referred from its drainage area.

All patients referred to the TAH melanoma MDT from 01 January 2015 to 31 December 2017 with a diagnostic biopsy showing CMM were considered. The cohort included male and female patients $>18$ years old with clinical stage (c Stage) T1b-T4b N0-3 M0-1a melanoma.

Patient records that had one or more exclusion criteria were omitted from further data analysis. Patients excluded from the study were:

- Those with uveal/mucosal melanoma

- T1a CMM or melanoma in situ

- Cutaneous melanoma undergoing primary non-surgical treatment

- Incomplete clinical records
- Patients with a second cancer diagnosis within 5 years of their melanoma diagnosis (except for localised squamous or basal cell carcinoma of the skin)

Hard copy clinical patient records were used in conjunction with records from other available electronic databases such as the hospital Enterprise Content Management (ECM) system, the National Health Laboratory System (NHLS) and the Picture Archiving and Communication system (PACS).

Demographic data, clinicopathological data of the tumour and institutional and clinical data were recorded. The primary variable was the interval between the date of biopsy and definitive surgery, known as the SI at TAH, measured in weeks. The factors affecting the SI were ascertained, and it was recorded whether any of these factors caused delays in referral for definitive surgical management.

The following clinical covariates included: age at diagnosis, sex, race (clinician-assigned ethnicity), comorbidities and anatomical site. We recorded the residential address for each patient and analysed the distance from the hospital in kilometres. The patients were divided as living within the metropolitan (metro) area or not. Tumour data included: Clinical tumour stage (c T Stage), nodal stage and metastatic stage (TNM). American Joint Committee on Cancer (AJCC) c Stage groups were allocated for each patient. ${ }^{9}$ Histological features such as melanoma subtype, Breslow thickness and ulceration were recorded.

Other institutional and clinical variables recorded were: the speciality of the physician performing the diagnostic biopsy, the type of initial biopsy (incisional vs. excisional), the place where the surgery was performed and whether a Positron emission tomography with computed tomography (PET-CT) scan scan or sentinel lymph node biopsy (SLNB) was done. We also noted the type of definitive surgery done for each patient and noted the pathological tumour stage (pT Stage) and the final pathological stage (p Stage) group. By recording the date of diagnostic biopsy, date of first MDT and date of definitive surgery, we calculated time from biopsy to MDT in weeks, time from MDT to surgery in weeks and time from biopsy to surgery (the SI) in weeks.

Survival was defined as the time from histological diagnosis to the date of death or last follow-up for living patients. Dates of death were obtained from hospital records and the South African Medical Research Council National Population Register.

Descriptive statistics were used to analyse and report the prevalence of individual variables. Time was analysed as a continuous outcome, and non-parametric tests were used to compare the SI and descriptive factors which were characterised into groups. 
Survival outcomes in months from surgery were defined as alive or dead at the time of data censoring after a median follow-up period of 31 months from primary surgery.

Kaplan-Meier curves were used to estimate overall and 2-year survival. Log-rank tests were used to compare time to mortality between groups. A $p$-value $<0.05$ was considered as statistically significant.

\section{Ethical considerations}

Ethics approval was obtained from the University Health Research Ethics Committee, Stellenbosch University (ethics reference number $S 17 / 10 / 243$ ) and facility permission obtained from the regions Department of Health South Africa.

\section{Results}

\section{Patient and tumour characteristics}

A total of 40 patients met the inclusion criteria. Demographic and tumour data are illustrated in Table 1. The cohort consisted mainly of female patients $(52.5 \%)$. The median age was 59 years (range 25-82 years). Most patients were Caucasian $(82.5 \%)$. Thirteen patients had two or more other comorbidities. Sixty five percent of patients lived in the Cape metro area. Regarding distance from the hospital, most patients (95\%) lived within $200 \mathrm{~km}$ of TAH.

The most common primary melanoma site was the trunk and lower limb (35.0\% each), followed by the head and neck area $(15.0 \%)$ and the upper limbs (12.5\%). One patient $(2.5 \%)$ presented with nodal disease only. Patients mainly presented with thick tumours, up to $40 \%$ being a cT3 and $37.5 \%$, a cT4 supported by the data showing a median Breslow of $3.9 \mathrm{~mm}$ (range $0.5 \mathrm{~mm}-15 \mathrm{~mm}$ ) and ulceration in $57.5 \%$ of patients. Besides, $62.5 \%$ of patients were staged as c Stage II on presentation. However, after primary surgery, only $45 \%$ of patients were still classified as $\mathrm{p}$ Stage II. This was mainly because of eight patients who were initially at c Stage II but were upstaged after SLNB to p Stage III. Two patients were down-staged after primary surgery.

Analysis of the histological subtypes revealed 35 patients with non-acral melanoma (superficial spreading, nodular and type not otherwise specified) and five patients with acral melanoma. Excisional biopsies were done in most patients $(n=27)$.

\section{Institutional and clinical data}

It was noted that non-dermatologists were performing up to $70 \%$ of initial biopsies. Although most diagnostic biopsies were done at private general practitioners and district hospitals, $85 \%$ of patients had primary surgery at TAH (Table 2). This can be because $50 \%$ of cases had an indication for SLNB, done only at a tertiary hospital level. Fluorodeoxyglucose $\left({ }^{18} \mathrm{~F}\right)$ (FDG) and Positron emission tomography (PET-CT) scans are routinely used for staging in
TABLE 1: Patient and tumour characteristics.

\begin{tabular}{|c|c|c|c|}
\hline Variable $(n=40)$ & $n$ & $\%$ & Median \\
\hline \multicolumn{4}{|l|}{ Gender } \\
\hline Male & 47.5 & 19.0 & - \\
\hline Female & 52.5 & 21.0 & \\
\hline Age (median range) (years) & 59.0 & & $25-82$ \\
\hline \multicolumn{4}{|l|}{ Ethnicity } \\
\hline White people & 33 & 82.5 & - \\
\hline Black people & 6 & 15.0 & \\
\hline Mixed race people & 1 & 2.5 & \\
\hline Comorbidity ( $\geq 2$ ) & 13 & 32.5 & - \\
\hline Urban patients & 26 & 65.0 & - \\
\hline $\begin{array}{l}\text { Distance from residence to TAH } \\
\text { (median range) }(\mathrm{km})\end{array}$ & 17 & - & $2-304$ \\
\hline \multicolumn{4}{|l|}{ Primary sites } \\
\hline Head and neck & 6 & 15.0 & - \\
\hline Trunk & 14 & 35.0 & - \\
\hline Upper limb & 5 & 12.5 & - \\
\hline Lower limb & 14 & 35.0 & - \\
\hline Nodal only & 1 & 2.5 & - \\
\hline \multicolumn{4}{|l|}{ c T Stage } \\
\hline Tx & 2 & 5.0 & - \\
\hline $\mathrm{T} 1$ & 4 & 20.0 & - \\
\hline $\mathrm{T} 2$ & 5 & 12.5 & - \\
\hline T3 & 16 & 40.0 & - \\
\hline T4 & 15 & 37.5 & - \\
\hline \multicolumn{4}{|l|}{ c Stage group } \\
\hline Stage 1 & 8 & 20.0 & - \\
\hline Stage 2 & 25 & 62.5 & - \\
\hline Stage 3 & 5 & 12.5 & - \\
\hline Stage 4 & 2 & 5.0 & - \\
\hline \multicolumn{4}{|l|}{ p Stage group } \\
\hline Stage 1 & 8 & 20.0 & - \\
\hline Stage 2 & 18 & 45.0 & - \\
\hline Stage 3 & 13 & 32.5 & - \\
\hline Stage 4 & 1 & 2.5 & - \\
\hline Ulceration present & 23 & 57.5 & - \\
\hline Breslow thickness (median) (mm) & 3.9 & & $0.5-15.0$ \\
\hline \multicolumn{4}{|l|}{ Histology } \\
\hline Non-acral & 35 & 87.5 & - \\
\hline Acral & 5 & 12.5 & \\
\hline \multicolumn{4}{|l|}{ Biopsy Type } \\
\hline Incisional & 13 & 32.5 & - \\
\hline Excisional & 27 & 67.5 & - \\
\hline
\end{tabular}

Note: Stages were assigned using the American Joint Committee on Cancer (AJCC), 7th edition.

TAH, Tygerberg Academic Hospital; c T Stage, clinical tumour stage; c Stage, clinical stage; p Stage, pathological stage.

Stage III and above CMM. Positron emission tomography with computed tomography scans were indicated before surgery in $52.5 \%$ of cases.

\section{Surgical interval}

The median SI was 13.5 weeks (range 0.71-39 weeks), with a mean of 13.94 weeks as depicted in Figure 1. Forty-five percent of the patients had their surgery in less than 12 weeks.

To determine the influence of management, sub-intervals on the SI, that is, the times from biopsy to MDT and time from MDT to surgery, were determined and calculated as a median of 5.5 weeks and 7.5 weeks, respectively. 
TABLE 2: Institutional and clinical data.

\begin{tabular}{lcc}
\hline Variable & $\boldsymbol{N}$ & $\mathbf{\%}$ \\
\hline Place of surgery & 34 & \\
TAH & 6 & 85.0 \\
Non-TAH & 20 & 15.0 \\
SLNB & 21 & 50.0 \\
PET-CT scan & & 52.5 \\
\hline
\end{tabular}

TAH, Tygerberg Academic Hospital; SLNB, sentinel lymph node biopsy; Fluorodeoxyglucose ( $\left.\left.{ }^{18} \mathrm{~F}\right] \mathrm{FDG}\right) ;$ PET-CT, positron emission tomography with computed tomography scan.

\section{Association between descriptive factors and surgical interval}

The descriptive factors were further grouped and analysed, then compared to the SI to elucidate factors associated with delays in the management of CMM. For patients who lived in the metro area, the median SI was 11.14 weeks ( $p$-value 0.04). For patients living outside the metro area, the median SI was significantly longer at 15.43 weeks. There was a significant association between the $\mathrm{p}$ Stage group and the SI ( $p$-value 0.01 ), where the SI was noted to be longer for patients with stage 3 and 4 disease.

Patient demographics such as age, gender, co-morbidities or ethnicity were not significantly associated with the SI.

\section{Overall survival}

After a median follow-up of 31 months, the estimated 2 year OS for the entire cohort was $77 \%$. Thirteen patients $(32.5 \%)$ were deceased, with progressive melanoma noted as the cause of death in 10 (25\%). The median OS was unreached (Figure 2). Overall survival differed significantly by pathological Stage group (Figure 3).

In this cohort, there was no significant association between the OS and SI.

\section{Discussion}

Timeous surgery for localised cancer affects the quality of healthcare outcomes. However, studies investigating the SI for resectable melanoma in South Africa have not been performed. This study aimed to determine the magnitude of the SI for cutaneous melanoma in a South African context.

Most developed countries have seen a rise in the incidence of and mortality from invasive melanoma in the past two decades. ${ }^{10}$ Tod et al. performed a retrospective review of data captured by the National Cancer Registry (NCR) of South Africa (SA) for 2005-2013. Eleven thousand seven hundred forty-eight melanoma cases were reported to the NCR, with the overall incidence for SA being 2.7 per $100000 .^{11}$

In South Africa (SA), data on the incidence of melanoma are limited or outdated as most studies have focused on a single population group or a single province which makes comparisons of studies difficult. Western Cape data showed high incidences in Caucasians (33.5 per 100000 in females

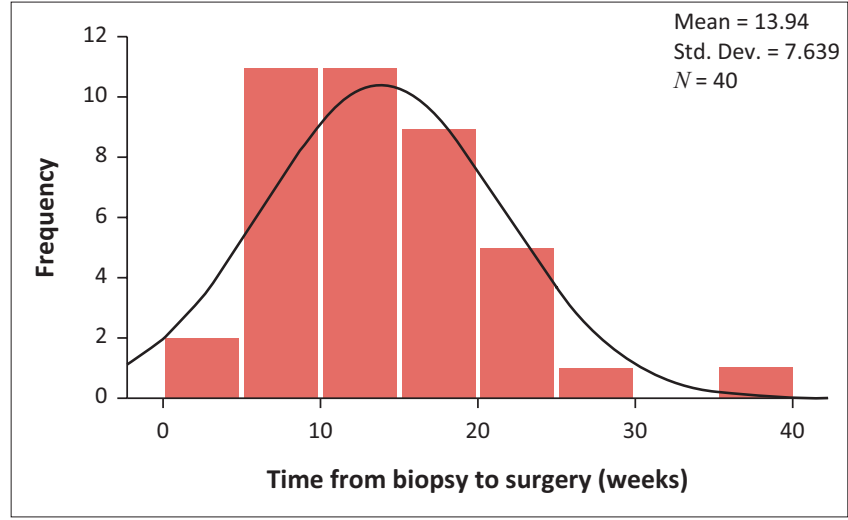

Std. Dev., standard deviation.

FIGURE 1: Mean time from biopsy to surgery.

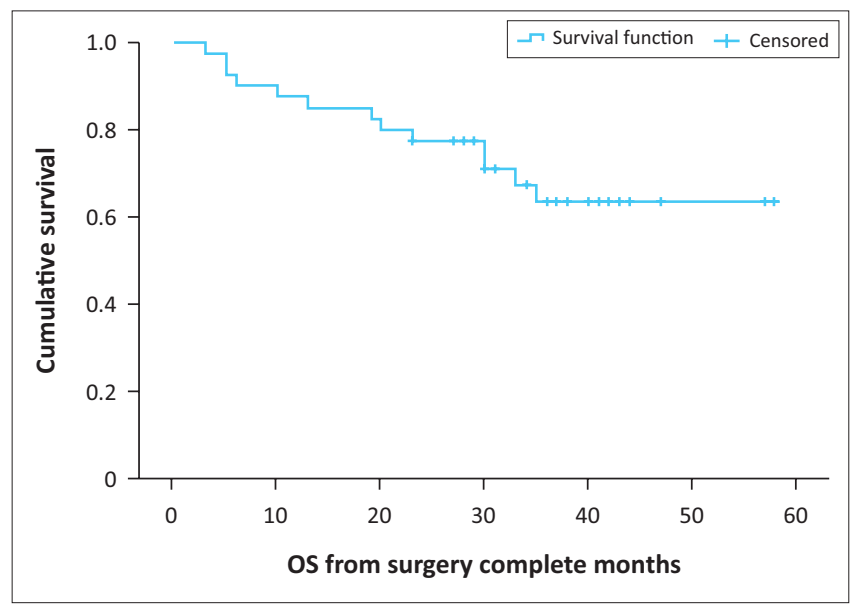

OS, overall survival.

FIGURE 2: Overall survival.

and 36.9 per 100000 in males for 2001-2003), ${ }^{12}$ whereas a study conducted in the Northern Cape found incidence rates for 2008-2012 of < 20 per 100000 for white people. ${ }^{13}$ The majority of our cohort patients were Caucasian (82.5\%) which does not reflect ethnic distribution in the Western Cape. This does, however, correlate with the ethnic distribution in the study by Tod et al. ${ }^{10}$

The cohort saw primarily Stage 2 tumours on presentation with a median Breslow of $3.9 \mathrm{~mm}$ and ulceration present in 57.5\%; however individual histopathological features were not significantly associated with the SI. Most international data looked at less advanced melanoma with a Breslow range of $0.9 \mathrm{~mm}-2.1 \mathrm{~mm}$ and failed to show any significant association with the SI.6,8 A review of skin cancer in the Northern Cape of South Africa also saw more advanced CMMs at diagnosis in the public sector with a median Breslow of $4.1 \mathrm{~mm} .{ }^{6}$ Socioeconomic factors influence disease outcomes in CMM, and less affluent groups generally have more advanced disease and lower survival rates. ${ }^{14}$ Besides, longer waiting times in the public sector may impact late diagnosis. Tumour thickness is an indicator of the effectiveness of early detection of melanomas. There is a focus on earlier detection in many countries; therefore, many thinner and in situ melanomas are being discovered..$^{13}$ 


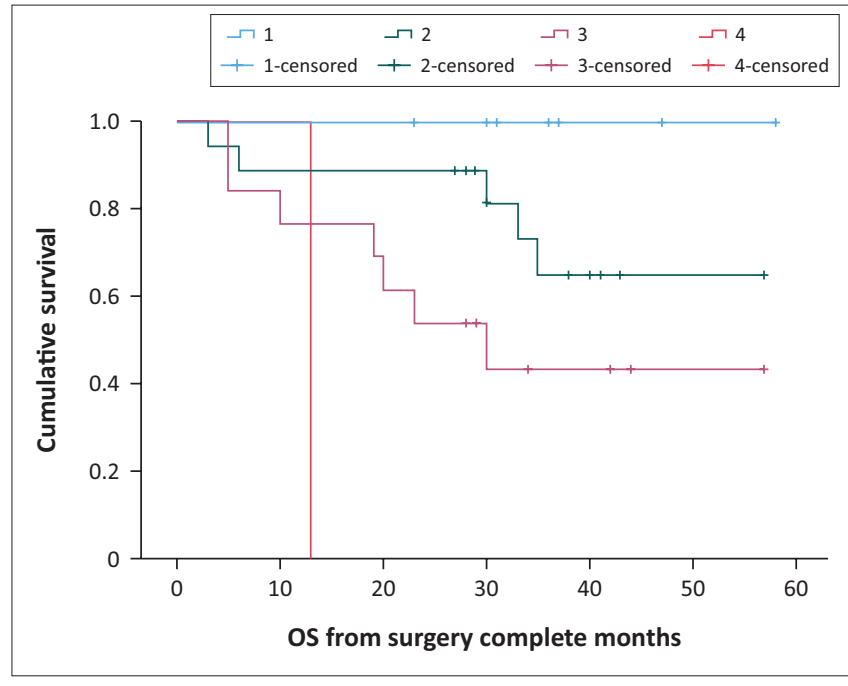

OS, overall survival.

FIGURE 3: Overall survival by pathological Stage group.

Acral melanomas are often diagnosed at an advanced stage. They are a rare subtype of CMM and usually account for only $1 \%-7 \%$ of all CMMs diagnosed, disproportionately affecting skin of colour. ${ }^{15}$ Acral melanomas made up $12.5 \%$ of our cohort, and of the five cases of acral melanoma, only one occurred in a Caucasian.

Another factor that was compared with the SI was whether a patient had a PET-CT scan. In a study by Boland MR, ${ }^{6}$ the median SI in patients who had had any form of imaging performed was 36 days, which was not statistically significant. Nineteen percent of our cohort underwent a PET-CT scan before surgery; however, this was not significantly associated with the SI. With difficulties in resource-constrained public sectors, one would have expected a delay in SI whilst awaiting a specialised investigation such as PET-CT. This was possibly alleviated because TAH has easy access to the positron emission tomography (PET) centre, located within the same building. Additionally, strict protocols for when and how to use PET-CT scans and screening the request forms allow these necessary slots to be available without undue delay.

Surgical interval guidelines for CMM are not well defined, and very few studies have evaluated the SI and its effect on oncological outcomes. ${ }^{2}$ Limited studies suggest that definitive treatment should be offered timeously but does not need to be immediate, again illustrating the paucity of evidence regarding an optimal SI. ${ }^{8,7,16}$ Although the extrapolated international standard of care of 12 weeks was only met in $45 \%$ of this cohort, the SI of 13.5 weeks in this cohort is comparable to international standards. The Tuscany Tumour Institute recommendations suggest performing definitive surgery within 3 months from the biopsy. Cited in this study ${ }^{17}$ was a recommendation to perform definitive surgery within 12 weeks from diagnostic biopsy. ${ }^{17}$ Melanoma quality indicators developed between July 2014 and June 2015 by the Scottish National Cancer Quality Steering Group (NCQSG) included a specific recommendation for a SI of 12 weeks, based on clinical consensus and current best practice. ${ }^{16,18}$ In this study ${ }^{18}$, only $64.7 \%$ of melanoma patients had a wide local excision within 12 weeks of their diagnostic excision biopsy and targets were not met. A study by Lott et al. ${ }^{19}$ looked at the delay of surgery for melanoma amongst Medicare beneficiaries. The authors demonstrate that 1 in 5 patients with a new diagnosis of melanoma experiences delays in re-excision or definitive excision of longer than 1.5 months, and $8.1 \%$ of them experience delays of longer than 3 months. ${ }^{20}$

The timing of definitive surgery for melanoma is associated with many factors, some of which can be controlled and others not. One of the biggest challenges faced at TAH includes transportation of patients on government transport and its availability. This, in turn, leads to missed appointments, larger tumours on presentation and delays in treatment. Our study showed that patients who lived within the metro area had a median SI of 11 weeks $(p<0.04)$. There was a multitude of reasons for the delay in wide local excision in the Scottish NCQSG study. Some of the reasons identified delays in pathology reporting, MDT discussions, scheduling of surgical procedure, comorbidities and patient wishes. The longest delay was the waiting time for plastic clinic appointments. ${ }^{16,18}$ A retrospective study performed by Boland et al. reviewed 107 patients who had a DEB followed by a wide local excision over 1 year. Factors associated with a delayed SI were increased age, if a dermatologist performed the excision as opposed to a general surgeon and lesions of the head and neck. ${ }^{6}$ This contrasted with another study investigating the delay of surgery amongst Medicare beneficiaries which showed that patients undergoing biopsy and surgery by dermatologists had the lowest risk of delay. Age and comorbidities were also significant. ${ }^{19}$

A Scottish study aimed at establishing reasons for poor prognosis in British patients with melanoma and found specific points in the diagnostic timeline at which delays to treatment occurred. There were no significant surgical delays associated with the lesion's site, age or sex; however, the fear of surgery and lack of knowledge played a role. ${ }^{21}$ Because of the rich diversity of cultures and beliefs in the SA population, traditional beliefs regarding surgery, consultation with family and elders may also play a role in delays, but this was not addressed in this study. The review of melanoma in the Northern Cape of South Africa postulated that longer waiting times for appointment in the public sector might impact late diagnosis. ${ }^{22}$

Tadiparthi et al. examined the type of biopsies done for melanoma in 100 patients and found that a significant proportion was inadequate and inconsistent with melanoma guidelines. This can lead to a delay in diagnosis and subsequent definitive treatment. ${ }^{20}$ We examined the type of biopsy done in each patient; however, no significant association was found.

The effect of SIs on oncological outcomes is known for some types of cancer (rectal, colon, breast) and has 
existing guidelines. In breast cancer, definitive treatment is recommended within 12 weeks of presentation with localised disease and delays are associated with increased mortality. ${ }^{7,23}$ McKenna et al. showed no effect of SI on OS or disease-free survival (DFS). Carpenter et al. also concluded that there was no effect on OS or DFS. A few studies have found no evidence of any difference in survival outcome or recurrence related to the SI. ${ }^{7,8,17}$ In this study, there was no significant association found between the OS and SI. The estimated 2 year OS was 77\%, comparable to international published data when referencing the AJCC 8th Ed staging data.

Early diagnosis, however, is a key factor in improving the outcomes of cancer patients. ${ }^{24}$ The Breslow is unanimously considered the most important factor for survival in the early stages of disease. ${ }^{1}$ From this, we can appreciate the importance of early diagnosis. Our cohort unfortunately saw many thick (T3/4) tumours on presentation and from analysing Figure 3. One can appreciate the decrease in OS for more advanced $\mathrm{p}$ Stages. In addition to primary prevention, secondary prevention (i.e. early detection and screening) is likely to offer potential for reduction of mortality. Detecting melanoma during the radial locally invasive growth phase allows for surgical excision before occult micro metastatic spread. Increased public and professional awareness can reduce the proportion of thick lesions detected as demonstrated by many groups. ${ }^{25}$

\section{Study limitations}

The limited size of the study cohort reduces its statistical power and the generalisability of the results to other populations. The small sample size, unfortunately, correlates to the number of patients reviewed in the chosen time frame, who were referred to the institution's MDT, almost exclusively within the public sector.

This was a retrospective study of a selected group of patients referred to a single tertiary referral centre which may introduce selection bias.

\section{Study strengths}

Patients were followed up for a median of 29 months. Exact dates of death were known for all deceased patients, and cause of death was only unknown in one case. Being conducted in a tertiary referral hospital, the study adds reliable data to the limited knowledge on the optimal SI in a South African setting, as TAH manages most of the T1b and more advanced melanomas in its drainage area.

\section{Conclusion}

Even though the internationally recommended SI of 12 weeks was not met in the majority of cases, it was met for urban patients. Future research should contribute towards the definition of a validated SI guideline for resource-limited settings. Defining the recommended SI per stage group may also be particularly useful in resource-limited settings. Further studies should also explore the effects of surgical delays on oncological outcomes such as stage progression and survival.

\section{Acknowledgements Competing interests}

The authors declare that there are no financial or personal relationships that may have inappropriately influenced them in writing this article.

\section{Authors' contributions}

S.N. and H.B. conceived of the presented idea. S.N. developed and wrote the manuscript with assistance from H.B. T.E.M. performed statistical analyses. H.B. supervised this work. All authors discussed the results and contributed to the final manuscript.

\section{Funding information}

The authors received no financial support for the research, authorship and/or publication of this article.

\section{Data availability}

The authors confirm that the data supporting the findings of this study are available within the article and/or the supplementary materials.

\section{Disclaimer}

The views expressed in this article are the authors and coauthors own and not an official position of the institution.

\section{References}

1. Orzan OA, Șandru A, Jecan CR. Controversies in the diagnosis and treatment of early cutaneous melanoma. J Med Life [serial online]. 2015 [cited 2015 Apr 1]; $8(2): 132-141$. Available from: http://www.pubmedcentral.nih.gov/articlerender. fcgi?artid=4392104\&tool=pmcentrez\&rendertype=abstract

2. Huff LS, Chang CA, Thomas JF, et al. Defining an acceptable period of time from melanoma biopsy to excision. Dermatol Rep. 2012;4(1):e2. https://doi. org/10.4081/dr.2012.e2

3. Benson $A B$, Arnoletti JP, Bekaii-Saab $T$, et al. Clinical practice guidelines in oncology. J Natl Compr Canc Netw. 2011;9(11):1238-1290. https://doi. org/10.6004/jnccn.2011.0104

4. Gordon LG, Elliott TM, Wright CY, Deghaye N, Visser W. Modelling the healthcare costs of skin cancer in South Africa. BMC Health Serv Res. 2016;16(1):113. https:// doi.org/10.1186/s12913-016-1364-z

5. Follmann M, Schadendorf D, Kochs C, Buchberger B, Winter A, Wesselmann S Quality assurance for care of melanoma patients based on guideline-derived
quality indicators and certification. JDDG J der Dtsch Dermatologischen Gesellschaft. 2014;12(2):139-147. https://doi.org/10.1111/ddg.12238

6. Boland MR, Prichard RS, Bass GA, et al. Malignant melanoma: Factors affecting the surgical interval from excision biopsy to definitive surgical management. Ir J Med Sci. 2015;184(2):511-515. https://doi.org/10.1007/s11845-014-1157-5

7. Carpenter S, Pockaj B, Dueck A, et al. Factors influencing time between biopsy and definitive surgery for malignant melanoma: Do they impact clinical outcome? Am J Surg. 2008;196(6):834-843. https://doi.org/10.1016/j.amjsurg.2008.07.044

8. Mckenna DB, Lee RJ, Prescott RJ, Doherty VR. The time from diagnostic excision biopsy to wide local excision for primary cutaneous malignant melanoma may not affect patient survival. $\mathrm{Br} J$ Dermatol. 2002;147(1):48-54. https://doi. org/10.1046/j.1365-2133.2002.04815.x

9. Balch, Charles M, Mihm M. The revised melanoma staging system and the impact of mitotic rate. Ski Cancer Found. 2010;28(3):2-3.

10. Siegel RL, Miller KD, Jemal A. Cancer statistics, 2016. CA: Cancer J Clin. 2016;66(1):7-30. https://doi.org/10.3322/caac.21332 
11. Tod BM, Kellett PE, Singh E, Visser WI, Lombard CJ, Wright CY. The incidence of melanoma in South Africa: An exploratory analysis of national cancer registry data from 2005 to 2013 with a specific focus on melanoma in black Africans. S Afr Med J. 2019;109(4):246-253. https://doi.org/10.7196/SAMJ.2019.v109i4.13565

12. Jessop S, Stubbings H, Sayed R, Duncan-Smith J, Schneider J, Jordaan H. Regional clinical registry data show increased incidence of cutaneous melanoma in Cape Town. S Afr Med J. 2008;98(3):197-199.

13. Ansell OP, Barton J, Bull D, et al. Effect of rapid referral on thickness of melanomas: The neuropathy of the critically ill. 1986; 1985.

14. MacKie R, Hole D. Incidence and thickness of primary tumours and survival of patients with cutaneous malignant melanoma in relation to socioeconomic status. BrMed J.1996;312(7039):1125-1128. https://doi.org/10.1136/bmj.312.7039.1125

15. De Wet J, Tod B, Visser WI, Jordaan HF, Schneider JW. Clinical and pathological features of acral melanoma in a South African population: A retrospective study. Afr Med J. 2018;108(9):777-781. https://doi.org/10.7196/SAMJ.2018.v108i9.13435

16. Information Services Division Publication Report. Melanoma quality performance indicators. 2016; (June 2015)

17. Crocetti E, Caldarella A, Massi D, Sacchettini C, Amunni G, Borgognoni L. Indicators of the standard of care for melanoma: Tuscany data. Melanoma Res. 2013;23(4):283-289. https://doi.org/10.1097/CMR.0b013e328362916a

18. Taskforce SC, Scotland HI. Scottish cancer taskforce cutaneous melanoma clinical quality performance indicators. 2014; 2016(June).
19. Lott JP, Narayan D, Soulos PR, Aminawung J, Gross CP. Delay of surgery for melanoma among medicare beneficiaries. JAMA Dermatol. 2015:151(7):731-741. https://doi.org/10.1001/jamadermatol.2015.119

20. Tadiparthi S, Panchani S, Iqbal A. Biopsy for malignant melanoma - Are we following the guidelines? Ann R Coll Surg Engl. 2008;90(4):322-325. https://doi. org/10.1308/003588408X285856

21. Doherty VR, MacKie RM. Reasons for poor prognosis in British patients with cutaneous malignant melanoma. Br Med J. 1986;292(6526):987-989. https://doi. org/10.1136/bmj.292.6526.987

22. York $\mathrm{K}$, Dlova NC, Wright $\mathrm{CY}$, et al. Primary cutaneous malignancies in the Northern Cape province of South Africa: A retrospective histopathologica review. S Afr Med J. 2017;107(1):83-88. https://doi.org/10.7196/SAMJ.2016. v107.i1.10924

23. Richards MA, Smith P, Ramirez AJ, Fentiman IS, Rubens RD. The influence on survival of delay in the presentation and treatment of symptomatic breast cancer Br J Cancer. 1999; 79(56):85864. https://doi.org/10.1038/sj.bjc.6690137

24. Weller D, Vedsted P, Rubin G, et al. The Aarhus statement: Improving design and reporting of studies on early cancer diagnosis. Br J Cancer. 2012;106(7):1262-1267. https://doi.org/10.1038/bjc.2012.68

25. Grover R, Ross DA, McKelvie M, Morgan BD. Improving the early detection of malignant melanoma. Ann R Coll Surg Engl [serial online]. 1996 [cited 2015 Apr 1];
$78(3$ Pt 1):176-179. Available from: http://www.pubmedcentral.nih.gov/ articlerender.fcgi ?artid $=2502698 \&$ tool=pmcentrez\&rendertype $=$ abstract 